\title{
Balance between matrix metalloproteinases (MMP) and tissue inhibitors of metalloproteinases (TIMP) in the cervical mucus plug estimated by determination of free non-complexed TIMP Naja Becher*1, Merete Hein ${ }^{1}$, Niels Uldbjerg ${ }^{1}$ and Carl Christian Danielsen ${ }^{2}$
}

Address: ${ }^{1}$ Department of Obstetrics and Gynecology, Århus University Hospital, Skejby, DK-8200 Aarhus N, Denmark and ${ }^{2}$ Department of Connective Tissue Biology, Institute of Anatomy, University of Aarhus, DK-8000 Aarhus C, Denmark

Email: Naja Becher* - nbecher@dadlnet.dk; Merete Hein - merete.hein@dadlnet.dk; Niels Uldbjerg - uldbjerg@dadlnet.dk; Carl Christian Danielsen - ccd@ana.au.dk

* Corresponding author

Published: 30 September 2008

Reproductive Biology and Endocrinology 2008, 6:45 doi:10.1 186/1477-7827-6-45

This article is available from: http://www.rbej.com/content/6/I/45

(C) 2008 Becher et al; licensee BioMed Central Ltd.

This is an Open Access article distributed under the terms of the Creative Commons Attribution License (http://creativecommons.org/licenses/by/2.0), which permits unrestricted use, distribution, and reproduction in any medium, provided the original work is properly cited.
Received: 8 July 2008

Accepted: 30 September 2008

\begin{abstract}
Background: The cervical mucus plug (CMP) is a semi-solid structure with antibacterial properties positioned in the cervical canal during pregnancy. The CMP contains high concentrations of matrix metalloproteinase 8 and 9 (MMP-8, MMP-9) and tissue inhibitor of metalloproteinase I (TIMP-I). This indicates a potential to degrade extracellular matrix components depending on the balance between free non-complexed inhibitors and active enzymes.

Methods: Thirty-two CMPs collected during active labor at term were analyzed. Twelve CMPs were separated into a cellular and an extracellular/fluid phase and analyzed by gelatin and reverse zymography to reveal MMP and TIMP location. Twenty samples were homogenized, extracted and studied by the TIMP activity assay based on gelatin zymography. Enzyme-linked immunosorbent assay (ELISA) was used to determine TIMP-I, MMP-8 and MMP-9 protein concentrations, and gelatin and reverse zymography used to identify gelatinases and TIMPs, respectively. The Western blotting technique was applied for semi-quantification of alpha2-macroglobulin. An ELISA activity assay was used to detect MMP-8 and MMP-9 activity.
\end{abstract}

Results: ProMMP-2, proMMP-9, TIMP-I and TIMP-2 were almost exclusively located in the fluid phase compared to the cellular phase of the CMP. All the extracted samples contained MMP-8, MMP-9, TIMP-I, TIMP-2 and alpha2-macroglobulin. Free non-complexed TIMP was detected in all the samples analyzed by the TIMP activity assay and was associated with TIMP-I protein $(R=0.7 I$, $\mathrm{P}<0.00 \mathrm{I})$ and with the TIMP/MMP molar ratio (I.7 (I.I-2.5) (mean ( $95 \%$ confidence interval)) ( $R$ $=0.65, \mathrm{p}=0.002$ ). The ELISA activity assay showed no activity from MMP-8 or MMP-9.

Conclusion: Due to their extracellular location, potential proteolytic activity from neutrophilderived MMPs in the CMP could exert a biological impact on cervical dilatation and fetal membrane rupture at term. The functional TIMP activity assay, revealing excess non-complexed TIMP, and a molar inhibitor/enzyme ratio above unity, indicate that refined MMP control prevents CMPoriginated proteolytic activity in the surrounding tissue. 


\section{Background}

The cervical mucus plug (CMP) constitutes a strong barrier between the sterile uterine environment and the microbe-rich vagina and protects the fetus from ascending infection throughout pregnancy [1-3]. We now know that the CMP not only has antibacterial properties, but also contains high concentrations of the matrix metalloproteinases (MMPs) gelatinase A (MMP-2), gelatinase B (MMP9) and neutrophil collagenase (MMP-8) together with their specific inhibitors, the tissue inhibitors of metalloproteinases, TIMP-1 and TIMP-2 [4].

There exists an extensive quantity of literature on the subject of MMP and TIMP in human reproduction. We and others have linked MMP protein in the CMP [4], the cervix [5], the fetal membranes [6,7] and in amniotic fluid [8,9] to proteolytic activity in connection with term and preterm birth. It is tempting to explain cervical dilatation and membrane rupture solely on the basis of MMP protein detection and presumed MMP activity. But the functional balance between matrix-degrading enzymes and their inhibitors $[10,11]$ in the CMP and the heterogeneity of cervical matrix biology $[12,13]$, are fundamental to our understanding of complicated multi-faceted processes like term and preterm birth.

While working with MMP and TIMP in the cervical mucus plug, an important question arises: does the detection of MMP protein imply proteolytic activity? To answer this question, we have conducted the present study where biological enzyme availability, i.e. enzyme and inhibitor location in the CMP cellular or extracellular phases, will be analyzed and discussed. Furthermore we will describe the presence of free non-complexed MMP-inhibitors as an indicator of the functional inhibitor-enzyme balance using a TIMP activity assay.

Several traditional methods, such as enzyme-linked immunosorbent assay (ELISA), reverse zymography and western blotting, can detect TIMP protein [14]. Common to all these methods is that they, together with the free TIMP fraction, identify TIMP already complexed with MMPs. Determination of the fraction of non-complexed TIMP describes the physiological impact of MMP presence and for this purpose we have developed the TIMP activity assay which is able to estimate free TIMP.

The aim of the present work was to elucidate the proteolytic capacity of the MMPs previously detected in the CMP. This capacity depends upon three factors: whether or not the enzymes are released from the cells, their activation status and finally the degree of inhibition by endogenous inhibitors present in the fluid phase. The aim was approached by 1) localization of MMP and TIMP proteins to cell phase or fluid phase, 2) determination of the molar ratio of MMP inhibitors/MMPs, 3) quantification of active MMPs and 4) determination of non-complexed TIMP by development of a TIMP activity assay .

\section{Methods \\ Materials}

The 32 intact CMPs included in this study were shed spontaneously or manually retrieved during vaginal exploration (active labor, cervical dilatation from $2-10 \mathrm{~cm}$ ). The CMPs were either directly suspended in PBS (1:100) for determination of TIMP and MMP distribution between the cellular and the extracellular phases $(n=12$, suspension group) or frozen for later extraction $(n=20$, extraction group). The women were healthy and their mean age was 29 (range 16-36) years; they had all been through a normal pregnancy and delivered vaginally at term (gestational week 37-42). The Central Denmark Region Committee on Biomedical Research Ethics approved the project and informed consent was obtained from each patient. MMP-1EA (MMP-1 with no enzymatic activity but retained TIMP-binding capacity) and APMA-activated MMP-2 were kindly donated by Dr. Yoshifumi Itoh, The Kennedy Institute of Rheumatology, Imperial College, London.

\section{CMP suspension}

The samples $(\mathrm{n}=12)$ were diluted directly after collection without prior freezing. They were dissolved in PBS to a final 1:100 dilution using a mantoux syringe to ensure gentle suspension with no cell damage. After centrifugation (10 min, $\left.500 \mathrm{~g}, 4^{\circ} \mathrm{C}\right)$, the supernatant (fluid phase) and the precipitate (cell phase) were separated and the cell phase was re-suspended to the original 1:100 dilution. Cells were trypanblue dyed $(0.5 \% \mathrm{w} / \mathrm{w}$, Bie \& Berntsen A/ $S$, Rødovre, DK) and counted (Bürker-Türk cell counter chamber). To ensure that the suspension procedure did not cause cell damage and protein leakage, it was performed on two buffy coats containing fresh donor blood leukocyte fractions supplied by the blood bank. Samples were kept at $-80^{\circ} \mathrm{C}$ until analysis.

\section{CMP extraction}

Samples $(n=20)$ were extracted as previously described [4]. Briefly, mixed pulverized CMP was extracted in 50 $\mathrm{mM}$ Tris- $\mathrm{HCl}, 10 \mathrm{mM} \mathrm{CaCl}_{2}, 0.05 \%$ Brij 35 and $1 \mathrm{mM}$ PMSF, pH 7.4 two times overnight at $4{ }^{\circ} \mathrm{C}$ and once at $60^{\circ} \mathrm{C}$ (4 min). The three supernatants were pooled (final 1:100 dilution) and kept at $-80^{\circ} \mathrm{C}$ until analysis.

\section{Gelatin zymography}

Gelatin zymography was performed on CMP extractions, CMP suspensions and the two buffy coats. For the CMP suspensions and the buffy coats, the purpose was to analyze gelatinase activity in the fluid and the cell phases. From the identical fluid phase and cell phase dilutions 
(1:100), $0.5 \mu \mathrm{l}$ was loaded on a $10 \%$ gel. After electrophoresis performed as previously described [4], the proMMP-2 and proMMP-9 bands were scanned using a Shimadzu Cromato Scanner (CS-930, Shimadzu Corporation, Kyoto, Japan) for semi-quantification of gelatinolytic activity in the two phases.

\section{Reverse zymography}

Sixteen $\mu$ l of fluid and cell phase (CMP and buffy coat suspensions) or CMP extracts was mixed 1:1 with electrophoresis sample buffer and incubated for $15 \mathrm{~min}$ at $37^{\circ} \mathrm{C}$, and the gels (13\%) were loaded with $30 \mu \mathrm{l}$ of this mixture. TIMPs were identified by comparison with the following standards: TIMP-1 (4.0 ng/lane, Biogenesis, Pole, UK) and TIMP-2 (4.0 ng/lane, Sigma-Aldrich).

\section{TIMP activity assay}

This assay is built on the premise that active, but not inhibited, MMP-2 is entrapped by $\alpha_{2}$-macroglobulin $\left(\alpha_{2} M\right)$ [15]. By mixing CMP extract, APMA-activated MMP-2, and $\alpha_{2} \mathrm{M}$ prior to gelatin zymography, it is possible to conclude on the presence of free inhibitor in the sample since the MMP-2 (64 kDa) lysis band intensity is brought to reflect the concentration of free inhibitor in the $\mathrm{CMP}$ extracts. Figure 1 illustrates the basic principle of the assay.

The CMP extracts $(n=20)$ were all scanned for the presence of free MMP inhibitors by $\alpha_{2} \mathrm{M}$ MMP-2 entrapment. For each CMP, the wells were loaded with sample mixture corresponding to sample A) CMP $(2 \mu \mathrm{l})+\mathrm{MMP}-2(10 \mathrm{pg})$, and sample B) CMP $(2 \mu \mathrm{l})+\mathrm{MMP}-2(10 \mathrm{pg})+\alpha_{2} \mathrm{M}(0.5$ $\mu \mathrm{g})$. Sample A versus sample B demonstrate the change in MMP-2 $(64 \mathrm{kDa})$ lysis activity when $\alpha_{2} \mathrm{M}$ is added to a mixture of CMP and active MMP-2. Sample B) was prepared by simultaneous mixing of MMP- 2 and $\alpha_{2} \mathrm{M}$ (a gift from Lars Sottrup-Jensen, Department of Molecular Biology, University of Aarhus, Denmark) into the CMP extract. This mixture was incubated for $30 \mathrm{~min}$ at $37^{\circ} \mathrm{C}$. Before electrophoresis on gelatin mini-gels (7.5\%) performed as previously described [4], all samples were diluted 1:1 with electrophoresis sample buffer (4\% SDS, $20 \%$ glycerol, $0.1 \%$ bromophenol blue, $125 \mathrm{mM}$ Tris$\mathrm{HCl}, \mathrm{pH}$ 6.8) and incubated for $15 \mathrm{~min}$ at $37^{\circ} \mathrm{C}$. After electrophoresis and staining, the MMP-2 (64 kDa) lysis bands (representing the fraction of $10 \mathrm{pg}$ MMP-2 that the inhibitors in CMP were able to bind) were scanned and the lysis intensity was quantified and compared to the intensity of the standard containing 10 pg of active MMP2 alone.

Eight extracts were titrated at declining volumes (from 2.0 $\mu \mathrm{l}$ to $0.0625 \mu \mathrm{l})$ with constant MMP-2 $(25 \mathrm{pg})$ and $\alpha_{2} \mathrm{M}$ $(0.5 \mu \mathrm{g})$ concentrations. Moreover, to test whether the free inhibitor was indeed TIMP, other aliquots of these 8 sam- ples $(4 \mu \mathrm{l})$ were pre-incubated $15 \mathrm{~min}$ at room temperature with MMP-1EA (see Materials above) in excess (4 ng) in order to capture all free TIMP possibly present in the CMPs. Then, MMP-2 $(20 \mathrm{pg})$ and $\alpha_{2} \mathrm{M}(1 \mu \mathrm{g})$ were added simultaneously (total volume $10 \mu \mathrm{l}$ ). Half of the sample volume (mixed 1:1 with sample buffer) was loaded onto the gels and electrophoresed as described above. In this test experiment, all the free TIMP present in the CMP sample is captured by MMP-1EA and MMP-2 is not expected to give lysis band at $64 \mathrm{kDa}$ (i.e. all MMP-2 is entrapped by $\alpha_{2} M$ ) unless the CMP also contains non-TIMP inhibitors. Control experiments to test the functionality of the reagents comprised four wells: 1) $\left.\alpha_{2} \mathrm{M}(0.5 \mu \mathrm{g}), 2\right) \mathrm{MMP}-$ 2 (10 or $25 \mathrm{pg}), 3) \mathrm{MMP}-2+\alpha_{2} \mathrm{M}$, and, finally, 4) MMP2, TIMP-1 or TIMP-2 (50 pg) and $\alpha_{2} \mathrm{M}$ in combination.

\section{Enzyme-linked immunosorbent assay (ELISA)}

TIMP-1, MMP-8 and MMP-9 concentrations in the CMP extracts were determined in duplicate by means of a commercially available ELISA-kits based on a two-site sandwich format (GE Healthcare, Buckinghamshire, UK, product number RPN2611, RPN2619 and RPN2614, respectively). We have previously validated this assay system for use on CMP extracts [4].

ELISA activity assay (GE Healthcare) was performed on CMP extracts according to the manufacturer's instructions. Active MMP-8 (product number RPN2635) was measured in a range between $0.75-24.0 \mathrm{ng} / \mathrm{ml}$. The sensitivity was $1.2 \mathrm{ng} / \mathrm{ml}$, the inter-assay coefficient of variation (CV) was less than $19.0 \%$ and the intra-assay CV less than 13.2\%. Active MMP-9 (product number RPN2634) was measured in a range between $0.5-16.0 \mathrm{ng} / \mathrm{ml}$. The sensitivity was $0.125 \mathrm{ng} / \mathrm{ml}$, the inter-assay CV was less than $43 \%$ and intra-assay CV was less than $21.7 \%$.

\section{Western blotting}

Sixteen $\mu$ l of CMP extracts was mixed $1: 1$ with electrophoresis sample buffer and incubated for $15 \mathrm{~min}$ at $37^{\circ} \mathrm{C}$. A $6 \%$ gel was loaded with $30 \mu$ of sample mixture together with $\alpha_{2} \mathrm{M}$ standard (10 ng and $50 \mathrm{ng}$ ). Following electrophoresis under non-reducing conditions, proteins were blotted to a PVDF membrane using a wet transfer system (Bio-Rad Laboratories, California, USA) with cooling in $25 \mathrm{mM}$ Tris, $192 \mathrm{mM}$ glycine, $0.05 \%$ SDS, $\mathrm{pH} 8.3$ as transfer buffer. Membranes were blocked with $0.5 \%$ Tween 20/PBS for $0.5 \mathrm{~h}$ and then probed overnight at $37^{\circ} \mathrm{C}$ with primary antibody (rabbit anti-human $\alpha_{2} \mathrm{M}$ (A033, DAKO, Glostrup, DK) diluted 1:500 in 0.5\% skim milk powder/0.05\% Tween 20/PBS). For detection, we used alkaline phosphatase-conjugated goat anti-rabbit antibody (D487, DAKO) as secondary antibody and 5bromo-4-chloro-3-indolyl phosphate/nitroblue tetrazolium as substrate. The $\alpha_{2} \mathrm{M}$ content was semi-quantitated 

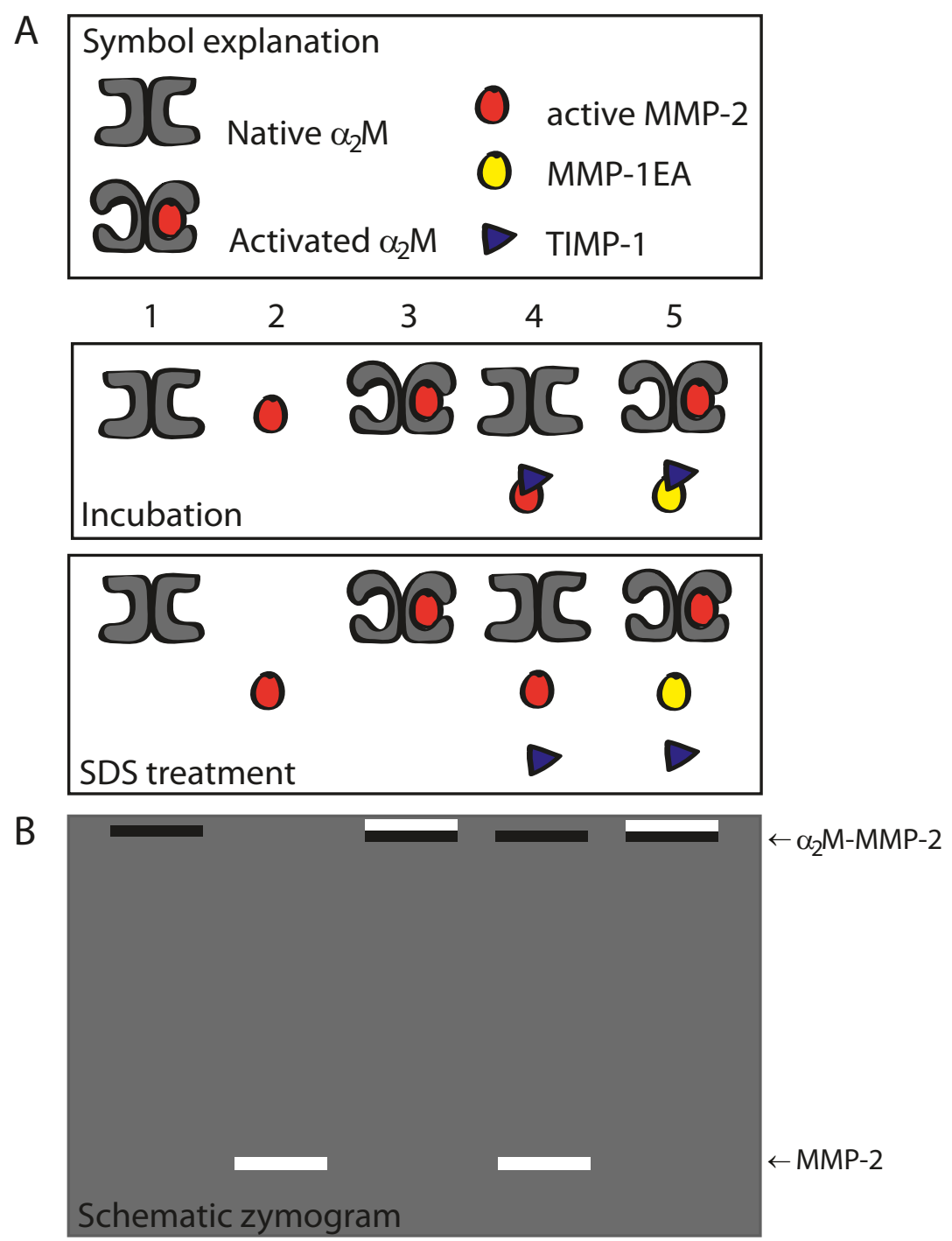

C

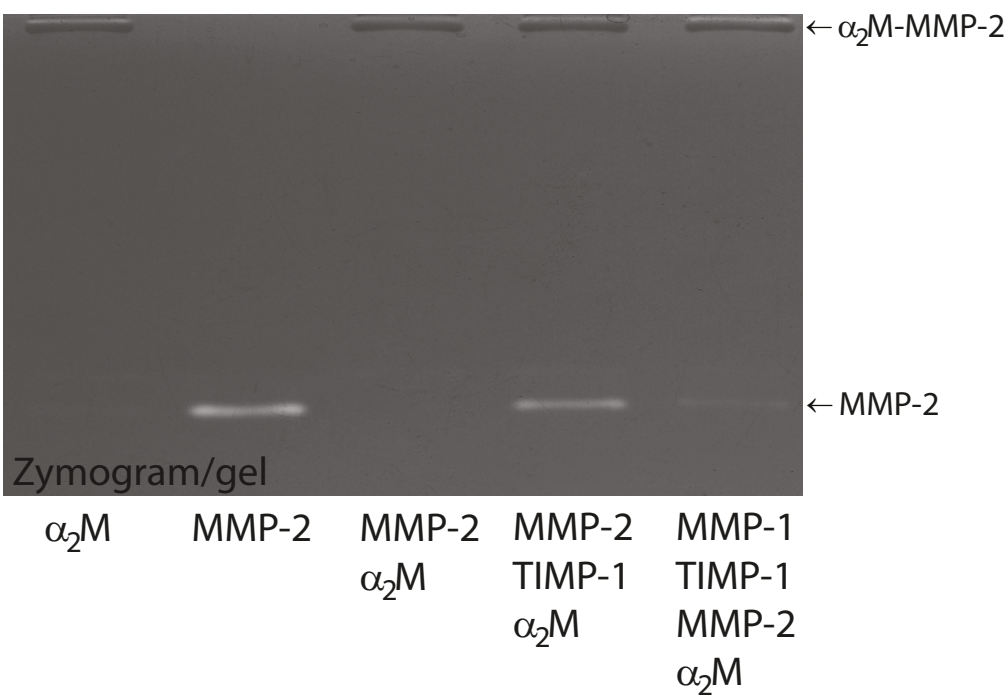

Figure I (see legend on next page) 
Figure I (see previous page)

Schematic illustration of the TIMP activity assay. The steps involved in TIMP activity assay during incubation, SDS treatment (inactivates MMPs, their reactivation is accomplished by SDS removal after electrophoresis) and zymography, respectively. (A): Symbol explanation and schematic illustration, (B): schematic zymogram and (C): a true zymogram. In lane I, $\alpha_{2} M$, represented in a monomeric form $(180 \mathrm{kDa})$, is visible as a high molecular weight protein band at the gel top. In lane 2, activity from active MMP-2 is visible as a lysis band at $64 \mathrm{kDa}$. Lane 3 illustrates the basic principle of the assay: active MMP-2 cleaves the $\alpha_{2} M$ bait region during incubation, is entrapped and stays covalently entrapped after SDS treatment. This is evident by a lysis band at the top of the zymogram together with $\alpha_{2}$ M. In lane 4, MMP-2 is pre-incubated with TIMP before the adding of $\alpha_{2} M$. During this pre-incubation TIMP will bind to MMP-2 and inhibit enzyme activity. When $\alpha_{2} M$ is thereafter added, MMP-2 cannot cleave the bait region. The following SDS treatment disassociates TIMP from MMP-2, and MMP-2 reappears on the zymogram as a $64 \mathrm{kDa}$ lysis band. In lane 5, TIMP-I is pre-incubated with MMP-IEA (TIMP-binding capacity but without enzymatic activity) which results in TIMP-I capture. When MMP-2 and $\alpha_{2}$ M are thereafter simultaneously added, MMP-2 is active and free to cleave the $\alpha_{2} M$ bait region resulting in entrapment. SDS disassociates the TIMP/MMP-IEA complex but the $\alpha_{2} M-$ MMP-2 complex stays intact. On the zymogram only the $\alpha_{2}$ M-MMP-2 complex is visible at the gel top.

in duplicate according to the standards using ImageJ software (NIH, USA).

\section{Statistics}

To obtain a normal distribution and an equal variance, the data were log-transformed when required. Linear regression analysis was used to investigate the relationship between the result of the TIMP activity assay and 1) molar TIMP-1 concentration, 2) the molar inhibitor/ enzyme ratios as well as the relationship between concentrations of MMP-8 and MMP-9. Protein concentration and molar ratio are presented as mean or geometric mean (95\% confidence interval (CI)). The Linear regression analysis was performed and the confidence intervals calculated using SigmaStat ${ }^{\circledR} 3.5$ (Systat). P-values below 0.05 were considered significant.

\section{Results}

CMPs from 32 women in active vaginal delivery were analyzed. The mean weight was $5.15 \mathrm{~g}$ (range 0.94-11.7 g). Twelve samples were directly diluted and separated into a fluid and a cell phase for determination of TIMP and MMP presence in the two phases. The remaining 20 samples were homogenized to obtain a representative measure of total MMP, TIMP and $\alpha_{2} \mathrm{M}$ protein.

\section{TIMP and MMP location}

We used reverse zymography and gelatin zymography to determine the presence of TIMP and MMP in the CMP fluid or cellular phases. ProMMP-2, proMMP-9, TIMP-1 and TIMP-2 were preferably located in the fluid phase (Figure 2A and 2B). On the gelatin zymograms, the ratio of proMMP-2 + proMMP-9 between the fluid phase and the cell phase was estimated by scanning of lysis band intensities and found to be 2.6 (1.4-3.8) (mean (95\% CI)). Control experiments with two buffy coats showed MMP and TIMP to be located in the cell phases, which confirmed that the suspension procedure did not damage the cells with consequential MMP release (Figure 2C and
2D). All the 12 suspended plugs contained numerous cells $\left(8.05 \times 10^{3}\left(3.52 \times 10^{3}-18.4 \times 10^{3}\right)\right.$ cells $/ m g$ CMP $)$ (geometric mean $(95 \% \mathrm{CI})$ ). The vast majority was recognized as leukocytes, but epithelial cells and possibly macrophages were also present. Five samples contained a few vital cells, maybe due to fresh blood contamination; otherwise all the cells were non-vital.

\section{Inhibitor-enzyme balance}

In Figure 3A the appearance and the functionality of the reagents used in the TIMP activity assay are demonstrated. This assay revealed free inhibitor in all 20 samples as illustrated by varying intensity of MMP-2 (64 kDa) lysis activity after $\alpha_{2} \mathrm{M}$ incubation (Figure $3 \mathrm{~B}$ ). After running a gel with 8 samples premixed with constant concentrations of active MMP-2 and $\alpha_{2} \mathrm{M}$ but with declining CMP extract concentrations, the MMP-2 (64 kDa) lysis bands were scanned. Lysis activity declined gradually with declining CMP concentrations, which demonstrated the inhibitory potential of each of the CMPs (Figure 3C). Capture of free TIMP by excess amount of MMP-1EA (Figure 3D) revealed that TIMP was not exclusively responsible for the inhibitor activity: the MMP-2 (64 kDa) lysis band was not completely abolished (Figure 3D, lane 9) and, therefore, not all the free inhibitor had been captured by MMP-1EA.

Nineteen samples contained TIMP-1 concentrations detectable by ELISA (mean 4.71 (3.10-7.16) (95\% CI) $\mathrm{ng} / \mathrm{mg} \mathrm{CMP}$ ) (molar concentration (fmol/mg CMP) shown in Figure 4). The TIMP-1 concentration varied considerably between the plugs (range $<1.25-29.0 \mathrm{ng} / \mathrm{mg}$ CMP). A high TIMP-1 molar concentration was associated with a large free inhibitor capacity as detected by the TIMP activity assay ( $\mathrm{R}=0.71, \mathrm{p}<0.001)$ (Figure $5 \mathrm{~A})$. The molar TIMP-1/(MMP-8 + MMP-9) ratio was above unity in 14 of 20 samples (mean $1.7(1.1-2.7)(95 \% \mathrm{CI}))$ and associated with free inhibitor capacity $(\mathrm{R}=0.65, \mathrm{p}=0.002)$ (Figure 5B). All 20 plugs revealed bands from both TIMP-1 (28.5 $\mathrm{kDa})$ and TIMP-2 (21.5 kDa) on the reverse zymograms 

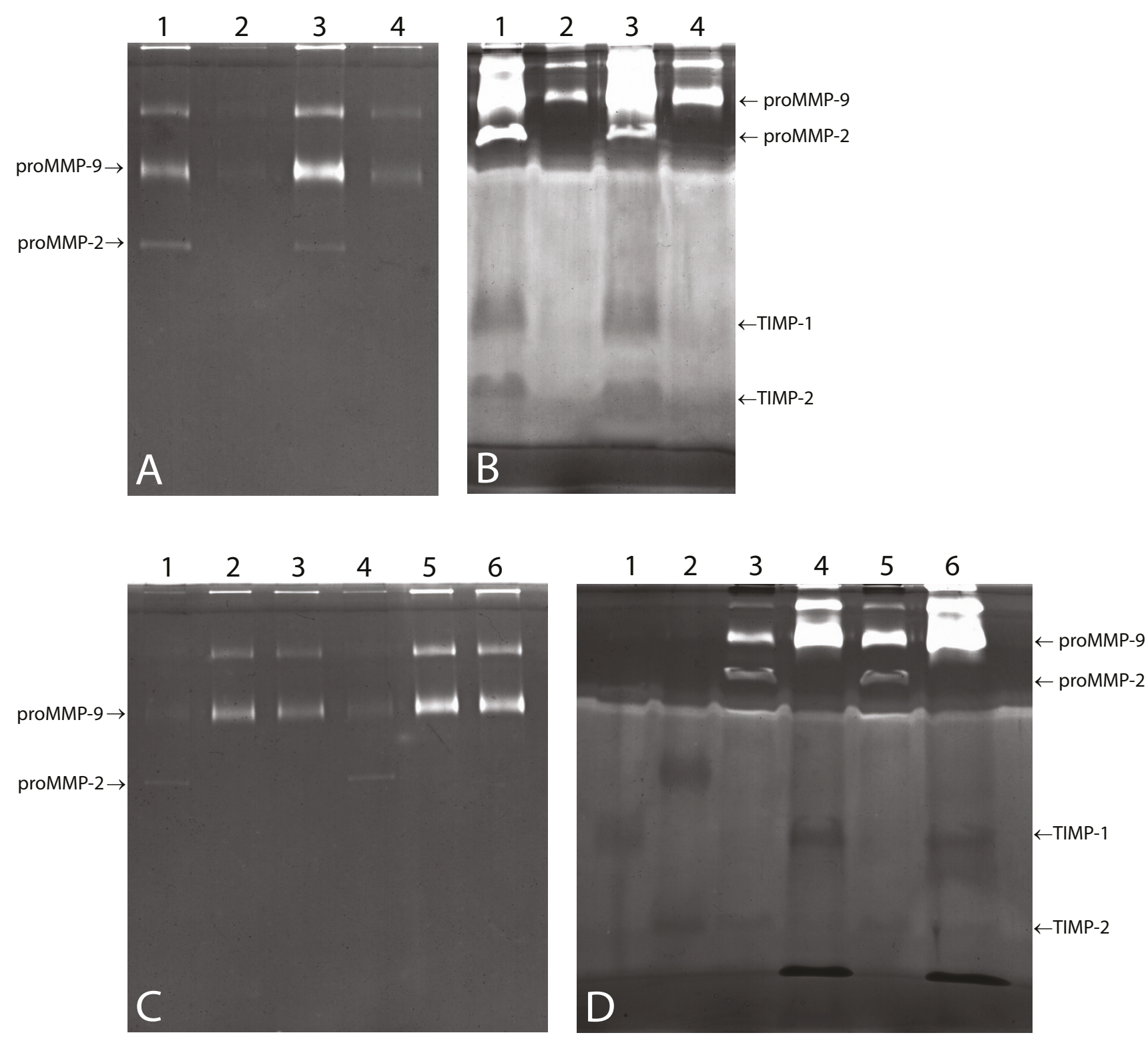

\section{Figure 2}

MMP and TIMP location. (A): gelatin zymography (I0\% gel) and (B): reverse zymography (I3\% gel) on the same two CMP suspensions; lanes I and 3: CMP fluid phases; lanes 2 and 4: CMP cell phases. Both enzymes and inhibitors are almost exclusively present in the fluid phases. (C): gelatin zymography performed on the two buffy coats; lanes I and 4: fluid phase, lanes 23 and 5-6: duplex of cellular phase. (D): Reverse zymography. Lane I: TIMP-I standard (4 ng, 28.5 kDa), lane 2: TIMP-2 standard (4 ng, 2I kDa), lanes 3 and 5: fluid phase. Lanes 4 and 6: cellular phase. For the buffy coats, TIMPs and MMPs are present in the cellular phase.

corresponding to the standards (not shown). Western blot proved $\alpha_{2} \mathrm{M}$ presence in all the samples (Figure 6) and the concentrations were semi-quantitatively estimated from band intensity (mean 34.5 (20.0-59.4) (95\% CI) ng/mg $\mathrm{CMP}$ ) (molar concentration (fmol/mg CMP) shown in Figure 4$)$. The molar ratio of total inhibitor (TIMP-1 + $\left.\alpha_{2} M\right) /(M M P-8+$ MMP-9) was above unity in 18 of 20 samples (mean $2.5(1.7-3.7)(95 \% \mathrm{CI})$ ) and associated with free inhibitor capacity $(\mathrm{R}=0.64, \mathrm{p}=0.002)$ (Figure 5C).

ELISA activity assays showed no active MMP-8 or active MMP-9. The mean protein concentration was for MMP-8 3.98 (2.84-5.59) (95\% CI) ng/mg CMP and for MMP-9 

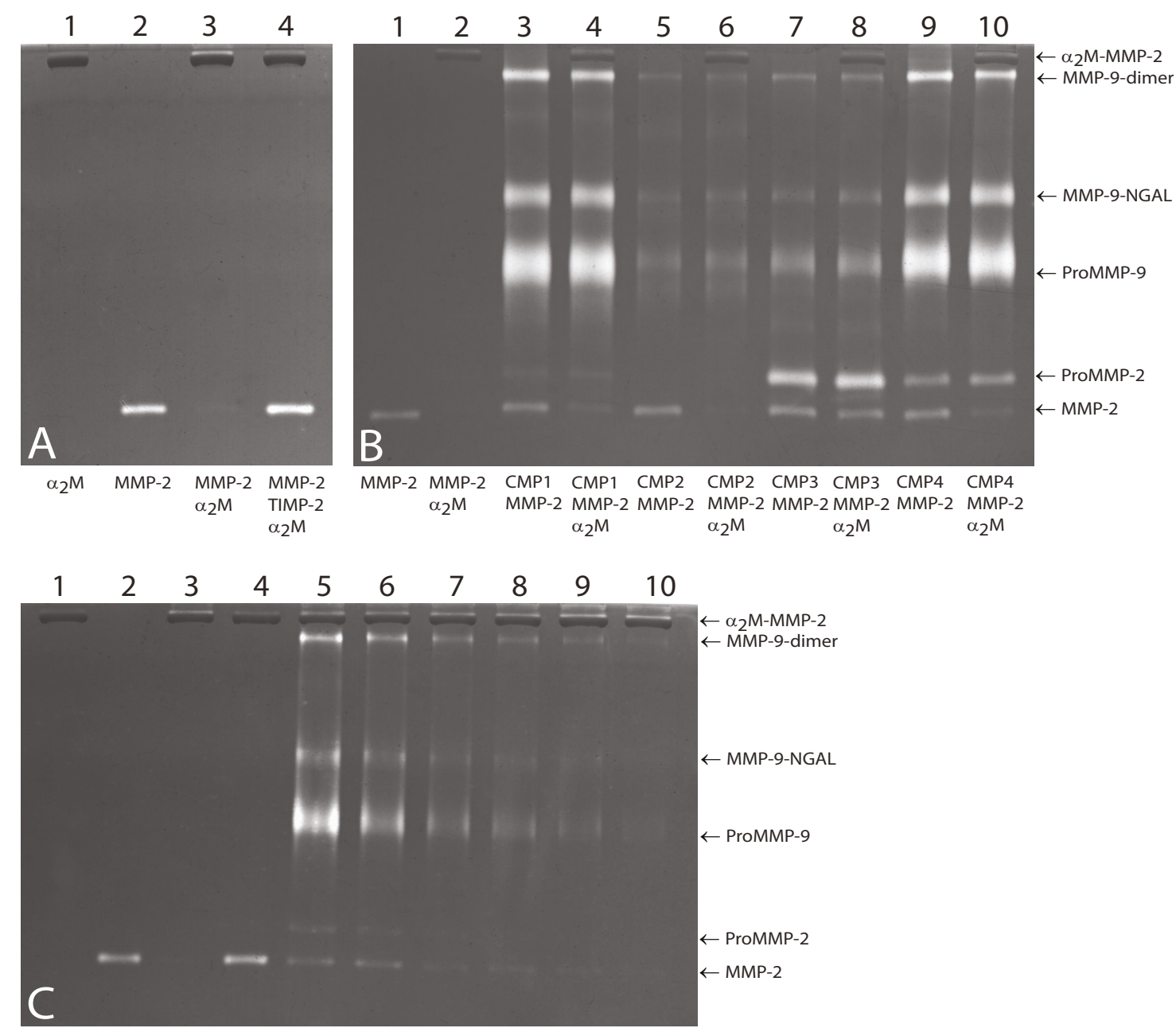

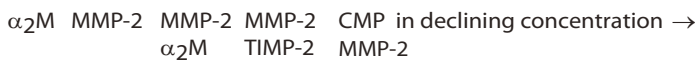
$\alpha_{2} \mathrm{M} \quad \alpha_{2} \mathrm{M}$

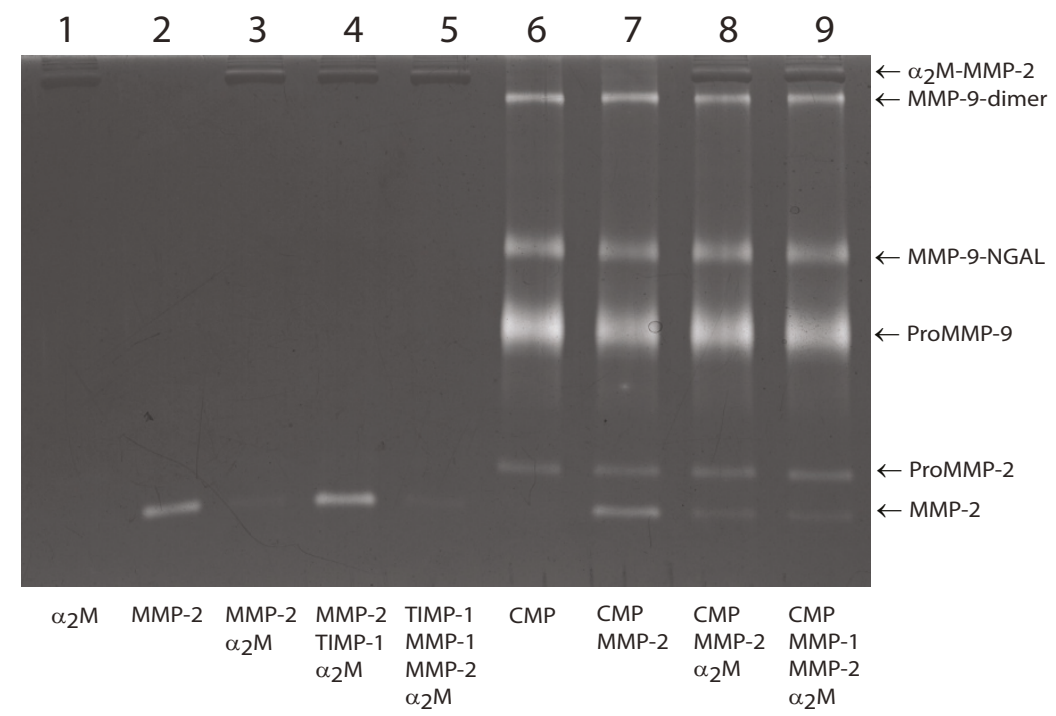

Figure 3 (see legend on next page) 
Figure 3 (see previous page)

Functional balance as illustrated by the TIMP activity assay. Panel (A) shows the standards as follows: lane I: $\alpha_{2} M(0.5$ $\mu \mathrm{g})$; lane 2: active MMP-2 (25 pg); lane 3: $\alpha_{2} \mathrm{M}+$ MMP-2; lane 4: MMP-2 + TIMP-2 $+\alpha_{2}$ M. (B): TIMP activity assay applied to four different CMPs: lane I: MMP-2 standard (I0 pg), lane 2: MMP-2 + $\alpha_{2}$ M. Lanes 3, 5,7 and 9: CMP (2 $\mu$ l) + MMP-2 (I0 pg). Lanes 4, 6, 8 and 10: CMP + MMP-2 + $\alpha_{2}$ M. MMP-2 (64 kDa) lysis bands suggest that an inhibitor in the sample prevented $\alpha_{2}$ M-MMP2 capture. (C): Titration of one inhibitor-positive plug. Lane I-4: controls. Lanes 5-10: MMP-2 (64 kDa) lysis activity declines gradually with declining CMP concentration $(2, \mathrm{I}, 0.5,0.25,0.125$ and $0.0625 \mu \mathrm{l})$. (D): MMP-IEA-TIMP capture experiment. Lane I-4: controls. Lane 5, MMP-IEA capture control: initial incubation of MMP-IEA (2 ng) and TIMP-I (50 pg) followed by simultaneous addition of MMP-2 (I0 pg) and a 2 M $(0.5 \mu \mathrm{g})$; MMP-IEA forms a complex with TIMP-I and allows $\alpha_{2}$ M-MMP-2 capture resulting in disappearance of MMP-2 $(64 \mathrm{kDa})$ lysis band. In lane 6-9, the MMP-IEA-TIMP capture experiment is applied to a CMP. Lane 6: CMP (2 $\mu$ l); lane 7: CMP + MMP-2 (10 pg); lane 8: CMP + MMP-2 + $\alpha_{2}$ M (0.5 $\mu$ g); lane 9: initially CMP and MMP-IEA (2 ng) followed by MMP-2 and $\alpha_{2}$ M. MMP-2 (64 kDa), proMMP-2 (72 kDa), proMMP-9 (92 kDa), MMP-9-NGAL complex (I $25 \mathrm{kDa})$, MMP-9 dimer (I84 kDa) and the $\alpha_{2}$ M-MMP-2 complexes are indicated. The analysis was performed on a $7.5 \%$ gel.

3.51 (2.65-4.65) (95\% CI) ng/mg CMP (molar concentration (fmol/mg CMP) shown in Figure 4). Total MMP-8 protein was directly proportional to total MMP-9 protein ( $\mathrm{R}=0.85, \mathrm{P}<0.001$ ) (not shown). Gelatin zymography revealed the characteristic lysis bands from MMP-9: bands at $184 \mathrm{kDa}$ (dimer), at $125 \mathrm{kDa}$ (MMP-9/NGAL complex), and at $92 \mathrm{kDa}$ (proMMP-9) in all 32 samples (not shown). Moreover, 16 of 32 samples revealed proMMP-2 lysis bands at $72 \mathrm{kDa}$, but there were no signs of active MMP-2 in any of the samples.

\section{Discussion}

With a combination of different methods we have tried to assess the proteolytic capacity of the MMPs previously

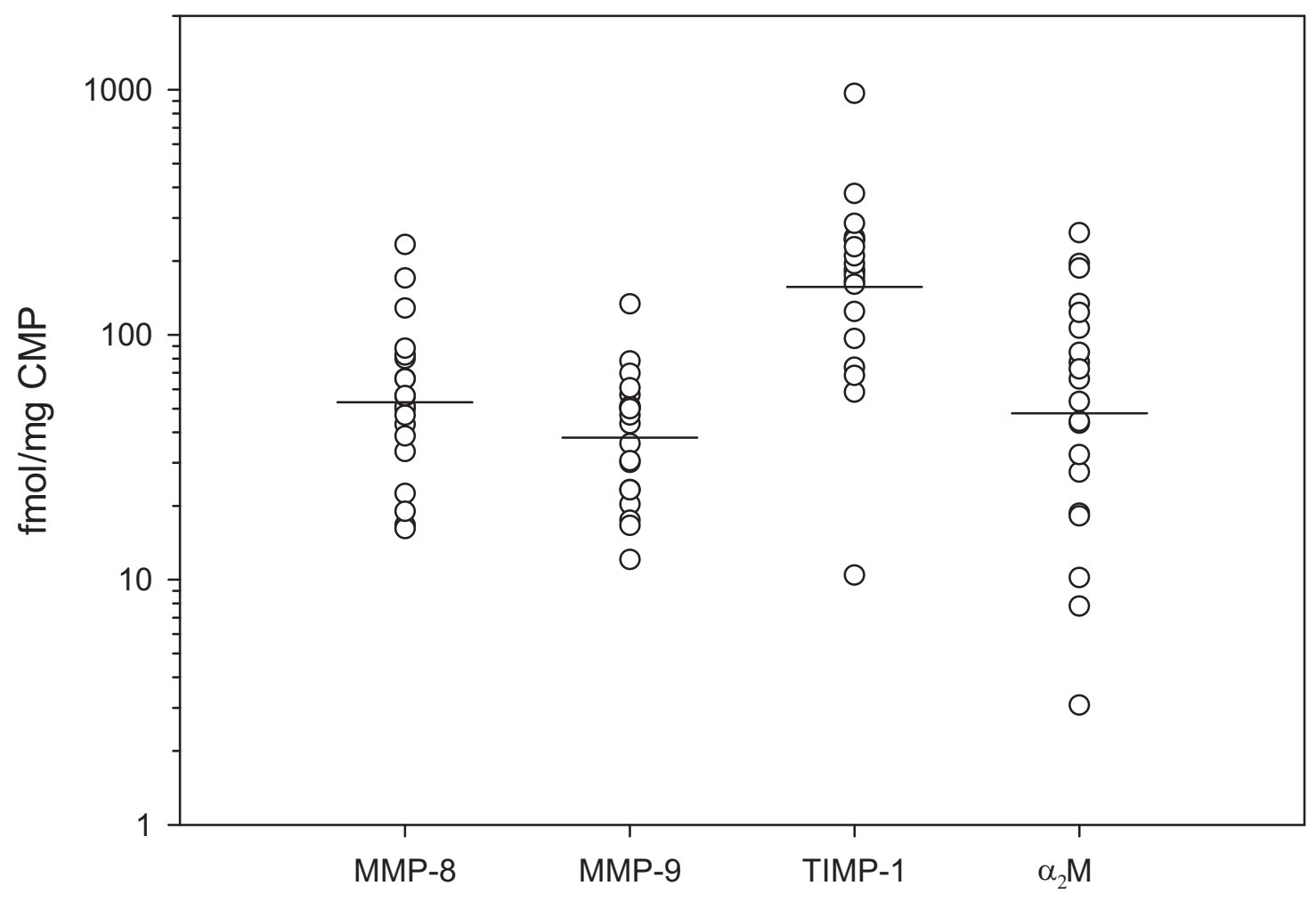

\section{Figure 4}

CMP content of enzymes and inhibitors. The molar concentrations of CMP MMPs and inhibitors in fmol/mg CMP. Every dot represents a CMP $(n=20)$ and geometric mean values are indicated. Note the log-scale. 

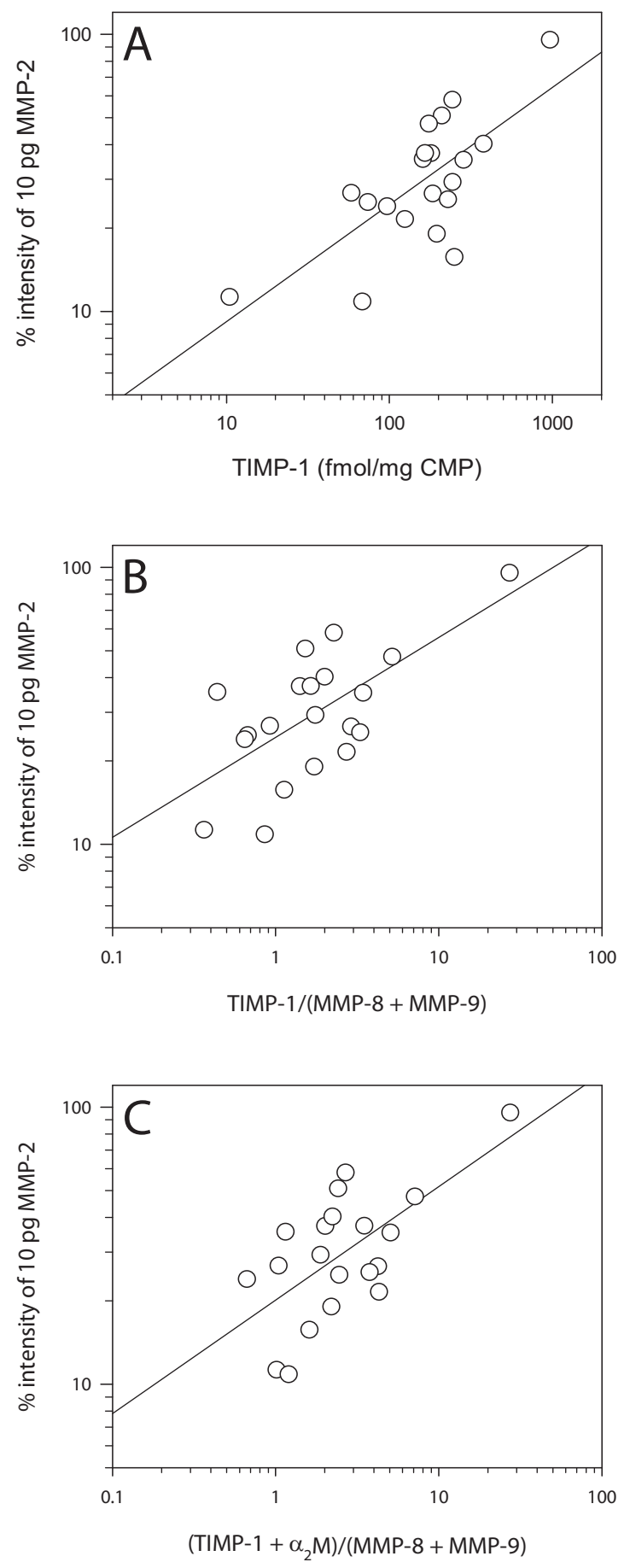

\section{Figure 5}

Regression analysis. Linear regression analysis of TIMP activity assay results versus (A) TIMP-I molar protein concentration $(R=0.7 I, p<0.00 I)$, (B) the TIMP-I/(MMP-8 + MMP-9) molar ratio $(R=0.65, p=0.002)$ and $(C)$ the $\left(T I M P-I+\alpha_{2} M\right) /(M M P-$ $8+$ MMP-9) molar ratio $(R=0.64, p=0.002)$. The MMP-2 $(64 \mathrm{kDa})$ lysis band intensities represent the fraction of $10 \mathrm{pg}$ MMP2 that the inhibitors in CMP were able to bind in percent of the intensity of the standard containing $10 \mathrm{pg}$ of active MMP-2 alone. 


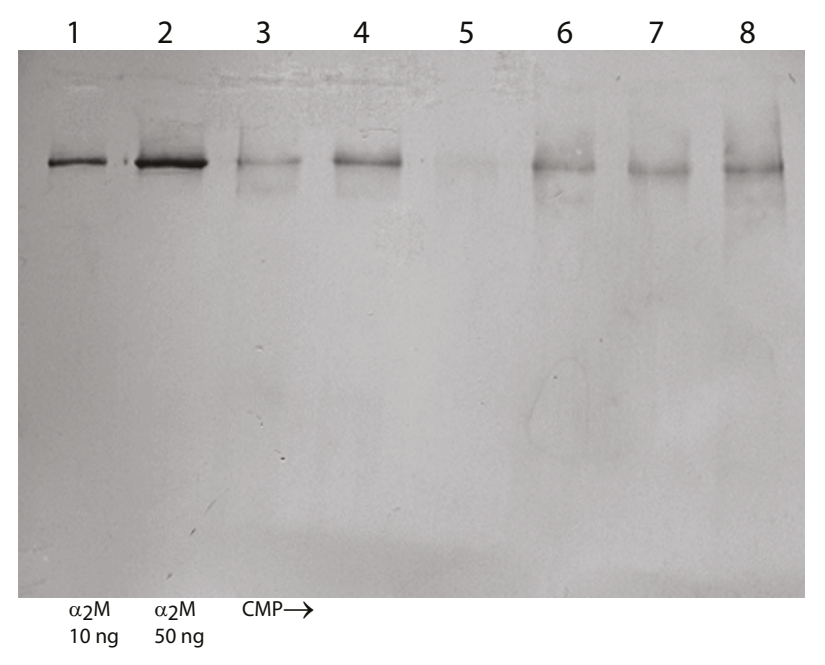

\section{Figure 6}

$\alpha_{2}$-macroglobulin Western blot. Lanes I and 2 contain standards, 10 and $50 \mathrm{ng} \alpha_{2} \mathrm{M}$ ( $180 \mathrm{kDa}$ monomer), respectively. Lanes 3-8 illustrate $\alpha_{2} M$ in 6 different CMP extracts.

detected in the cervical mucus plug at term of pregnancy. We mainly found both TIMP and MMP in the CMP fluid phase which could mean that they play a role in the biological function of the CMP. If the CMP, when positioned in the cervical canal during the course of pregnancy, can be compared to CMPs collected during active labor at term, the very high CMP MMP concentration might promote premature cervical softening and preterm premature rupture of the membranes (PPROM). The risk of these adverse effects is increased if the enzymes are both present in the fluid phase and represented in their active forms.

Granulocyte counting in mucus from the external cervical os reveals a significant increase in cells during the course of pregnancy with a further increase during active labor [16]. Microscopical observations performed on the mucus plug show cell clusters mainly in the part of the plug facing the vagina, and the predominant cell type observed here is the neutrophil leukocyte [3]. Therefore, the relatively high concentrations of MMP-8 and MMP-9 found in CMPs harvested during cervical dilatation probably derives from neutrophil invasion of the CMP as also evidenced by the CMP neutrophil count performed in this study. Indeed, the concentrations of MMP-8 and MMP-9 were correlated, which signifies a common cellular origin.

If the CMP MMP content primarily results from neutrophil leukocyte degranulation or apoptosis locally in the plug, the enzyme concentration may simply reflect CMP inflammation and antibacterial properties. The great inter-individual variation in enzyme concentrations among the CMPs analyzed could arise from differences in vaginal flora virulence and the consequential CMP antibacterial state of alert. MMP exudation from the dilating cervix undergoing extensive remodeling [17-19] could also contribute to CMP MMP, but to what degree is unknown. Additionally, recruitment of neutrophils caused by labor-induced inflammation may also add to the MMP level that we detect. We suggest that the neutrophil-derived MMPs in the CMP are expressed throughout pregnancy and reflect the main function of the CMP: to prevent preterm birth by protecting the fetus from ascending infection. As opposed to this clearly beneficial impact of neutrophil presence, the MMP content of neutrophil secretory granules may affect the cervix as well as the membranes in case of insufficient inhibition of the activated enzymes.

The TIMP activity assay is a most interesting method that builds on the basic principles of 1:1 stoichiometric MMPTIMP binding and $\alpha_{2} \mathrm{M}$ active proteinase entrapment, thus detecting free MMP inhibitors. We have applied this principle to the CMP and proved the functional inhibitorenzyme balance to be in favor of MMP-inhibition: all the CMP extracts analyzed contained free MMP inhibitor in various amounts. In spite of an extracellular location, the CMP MMPs are likely to play a minor role in the extensive cervical remodeling that occurs throughout an active birth process.

No active MMP- 8 or MMP-9 could be demonstrated by the ELISA activity assays. The most abundant MMPs in the CMP are not present in their active forms, but either in their pro-forms or complexed with TIMPs. The possibility exists that the concentrations of active MMPs are below detection limit of the kits used since the physiological concentrations of active MMPs in a tissue usually is very low. However, due to excess free TIMP, as demonstrated by the TIMP activity assay, this is not likely to be the case when considering the CMP. Should the pro-forms become activated, they will be immediately controlled by the free TIMP fraction. The formation of complexes between TIMPs and MMPs is rapid and strong; only restricted momentary pericellular activity [20,21], and not general proteolytic activity, is possible in a TIMP dominated environment $[22,23]$. The potential activity of MMP-8 and MMP-9 in the CMP seems to be tightly regulated by MMP-inhibitors which prevent uncontrolled $\mathrm{CMP}$-originated proteolytic activity in the surrounding tissues.

Efforts have been made to quantify the non-complexed free fraction of TIMP-1 in plasma by means of an immunoassay technique [24]. Concerning the CMP, the TIMP activity assay supplements immunodetection because it creates an in vitro interaction between the endogenous free 
CMP inhibitor and the added, active MMP-2. A functional reconstruction of highly complex interactions may allow generalization of the results to actual in vivo conditions. Control experiments with MMP-1EA capture of CMP TIMP-1 showed that the free inhibitor was not represented by TIMP-1 alone, but the association between the TIMP activity assay results and a high TIMP/MMP molar ratio is in favor of TIMP-1 being mainly responsible. A parallel analysis using both techniques would clarify the issue of non-complexed MMP inhibitor in the CMP and reveal if the free inhibitor found here is actually TIMP-1.

On the basis of a molar ratio, we found 1.7 times as much TIMP-1 in the CMP than MMP-8 and MMP-9 together which support the inhibitor superiority detected by the TIMP activity assay. When calculating the molar ratio, it must be taken into consideration that only TIMP-1, MMP8 and MMP-9 were measured. According to our previous studies [4], MMP-8 and MMP-9 are the two most abundant MMPs present in the CMP. We have measured MMP1 and MMP-7 in CMP extracts and found them to be undetectable. Neither MMP-3 could be detected (casein zymography, unpublished data). MMP-2, on the other hand, is measurable in low concentrations in extracted CMP, but was not included in the present study. However, other MMPs and MT-MMPs not yet characterized in the CMP may also be potential inhibitor targets. The reverse zymography showed TIMP-2 in the CMP samples, but nevertheless the TIMP-2 concentration is unquantifiable with the ELISA technique (levels below kit sensitivity (3.0 $\mathrm{ng} / \mathrm{ml})$ ). Thus, the molar inhibitor-enzyme ratio calculated on the basis of our results should be taken merely as an indication of an overall tendency towards inhibitor superiority.

The TIMP source may be cervical tissue exudation, endocervical gland secretion or secretion from vital or dead cells already present in the plug. The CMP contains predominantly neutrophil leukocytes but diverging opinions exist regarding their ability to produce TIMP-1. Opdenakke et al. state, that neutrophils are first-line defense leukocytes and that they do not produce gelatinase A (MMP-2) or TIMP-1 [25]. On the other hand, Triebel et al. have succeeded in isolating TIMP-1 from neutrophils, which presumably allow these cells to control their own MMP activity [26]. Concerning the CMP, the most likely option is that TIMP-1 is secreted from the endocervical glands together with the mucus that constitutes the plug.

If CMP TIMP originates from endocervical glands, the regulation of cervical TIMP synthesis and secretion must adjust to the highly individual MMP content to reach the refined enzymatic control that we describe. Such a regulatory mechanism is interesting in the context of preterm birth; if the cervix fails to modify TIMP levels to CMP MMP levels during the course of pregnancy, active proteolytic enzymes may cause cervical softening and/or PPROM leading to preterm delivery. Much effort has been displayed to use different inflammatory markers in cervical secretions to predict preterm labor [27]. A CMP sample is easy to obtain, and with TIMP and MMP preferably located in the fluid phase, these components can be reliably quantified. Future analysis of TIMP and MMP in CMP samples collected during the course of pregnancy may add to our understanding of term labor mechanisms and preterm labor pathophysiology.

\section{Conclusion}

In the present study we found that MMP and TIMP are located in the fluid rather than in the cellular phase of the $\mathrm{CMP}$ at term, that the CMP displays molar inhibitor excess, that the CMP contains no active MMP- 8 or MMP9 and finally, that the CMP contains free non-complexed MMP inhibitor which signifies a functional inhibitor superiority.

Our results suggest that the CMP, with neutrophil-derived MMPs available in the fluid phase, has a potential to display matrix degrading properties and therefore could play a role in the cervical dilatation and fetal membrane rupture at term. However, the TIMP activity assay, revealing free non-complexed TIMP, and the detection of a molar inhibitor/enzyme ratio above unity, indicate, that inhibitor control hinders CMP-originated proteolytic activity in the surrounding tissue. The regulation and biological importance of the refined CMP inhibitor-enzyme balance described here is yet to be established.

\section{Competing interests}

The authors declare that they have no competing interests.

\section{Authors' contributions}

$\mathrm{NB}, \mathrm{MH}, \mathrm{NU}$ and CCD have participated in the design of the study. The experiments were carried out by NB and CCD. NB, NU and CCD participated in the data analysis. The manuscript was written by NB. MH, NU and CCD assisted in revising it. All authors have read and approved the final manuscript.

\section{Acknowledgements}

We greatly appreciate the help of Dr. Yoshifumi Itoh, The Kennedy Institute of Rheumatology, Imperial College, London, for guidance while developing the TIMP activity assay. We are deeply grateful to the midwives at the labor wards at Aarhus University Hospital, Skejby, for their enthusiasm in collecting CMPs over the past several years. Special gratitude also goes to Eva K Mikkelsen for her excellent technical assistance. Grants from The Institute of Experimental Clinical Research, Aarhus University Hospital, Skejby, The Danish Medical Association Fond and a travel grant from the Faculty of Health Sciences, University of Aarhus supported this work. 


\section{References}

I. Hein M, Helmig R, Schonheyder HC, Ganz T, Uldbjerg N: An in vitro study of antibacterial properties of the cervical mucus plug in pregnancy. Am J Obstet Gynecol 200I, I 85:586-592.

2. Hein M, Valore EV, Helmig R, Uldbjerg N, Ganz T: Antimicrobial factors in the cervical mucus plug. Am J Obstet Gynecol 2002, I 87: | 37-| 44.

3. Hein M, Petersen AC, Helmig R, Uldbjerg N, Reinholdt : Immunoglobulin levels and phagocytes in the cervical mucus plug at term of pregnancy. Acta Obstet Gynecol Scand 2005, 84:734-742.

4. Becher N, Hein M, Danielsen CC, Uldbjerg N: Matrix metalloproteinases and their inhibitors in the cervical mucus plug at term of pregnancy. Am J Obstet Gynecol 2004, I9 I:1232-1239.

5. Sennström M, Brauner A, Byström B, Malmström A, Ekman G: Matrix metalloproteinase-8 correlates with the cervical ripening process in humans. Acta Obstet Gynecol Scand 2003, 82:904-9II.

6. El Khwad M, Stetzer B, Moore RM, Kumar D, Mercer B, Arikat S, Redline RW, Mansour JM, Moore J]: Term human fetal membranes have a weak zone overlying the lower uterine pole and cervix before onset of labor. Biol Reprod 2005, 72:720-726.

7. Arechavaleta-Velasco F, Marciano D, Díaz-Cueto L, Parry S: Matrix metalloproteinase-8 is expressed in human chorion during labor. Am J Obstet Gynecol 2004, 190:843-850.

8. Maymon E, Romero R, Pacora P, Gervasi MT, Gomez R, Edwin SS, Yoon $\mathrm{BH}$ : Evidence of in vivo differential bioavailability of the active forms of matrix metalloproteinases 9 and 2 in parturition, spontaneous rupture of membranes, and intra-amniotic infection. Am J Obstet Gynecol 2000, I 83:887-894.

9. Kim KW, Romero R, Park HS, Park CW, Shim SS, Jun JK, Yoon BH: $A$ rapid matrix metalloproteinase- 8 bedside test for the detection of intraamniotic inflammation in women with preterm premature rupture of membranes. Am J Obstet Gynecol 2007, 197:292-295.

10. Nagase H, Woessner JF Jr: Matrix metalloproteinases. J Biol Chem I999, 274:2|49|-2|494.

II. McCawley LJ, Matrisian LM: Matrix metalloproteinases: they're not just for matrix anymore! Curr Opin Cell Biol 200I, I 3:534-540.

12. Winkler $M$, Rath $W$ : Changes in the cervical extracellular matrix during pregnancy and parturition. J Perinat Med 1999, 27:45-60.

13. Word RA, Li XH, Hnat M, Carrick K: Dynamics of cervical remodeling during pregnancy and parturition: mechanisms and current concepts. Semin Reprod Med 2007, 25:69-79.

14. Troeberg L, Nagase H: Analysis of TIMP expression and activity. Methods Mol Med 2007, I35:25I-267.

15. Nagase H, Itoh $Y$, Binner S: Interaction of $\alpha_{2}$-macroglobulin with matrix metalloproteinases and its use for identification of their active forms. Ann N Y Acad Sci 1994, 732:294-302.

16. Luo L, Ibaragi T, Maeda M, Nozowa M, Kasahara T, Sakai M, Sasaki Y, Tanebe K, Saito S: Interleukin-8 levels and granulocyte counts in cervical mucus during pregnancy. Am J Reprod Immunol 2000, 43:78-84.

17. Junqueira LCU, Zugaib M, Montes GS, Toledo OMS, Krisztán RM, Shigihara KM: Morphologic and histochemical evidence for the occurrence of collagenolysis and for the role of neutrophilic polymorphonuclear leukocytes during cervical dilation. Am J Obstet Gynecol 1980, I 38:273-28I.

18. Osmers R, Rath W, Adelmann-Grill BC, Fittkow C, Kuloczik M, Tschesche Szeverényi M, Kuhn W: Origin of cervical collagenase during parturition. Am J Obstet Gynecol 1992, I 66: | 455- |460.

19. Stygar D, Wang H, Vladic YS, Ekman G, Eriksson H, Sahlin L: Increased level of matrix metalloproteinases 2 and 9 in the ripening process of the human cervix. Biol Reprod 2002, 67:889-894.

20. Owen CA, Hu Z, Lopez-Otin C, Shapiro SD: Membrane-bound matrix metalloproteinase-8 on activated polymorphonuclear cells is a potent, tissue inhibitor of metalloproteinaseresistant collagenase and serpinase. J Immunol 2004, | 72:779|-7803.

21. Owen CA, Hu Z, Barrick B, Shapiro SD: Inducible expression of tissue inhibitor of metalloproteinases-resistant matrix metalloproteinase-9 on the cell surface of neutrophils. Am J Respir Cell Mol Biol 2003, 29:283-294.
22. Bode W, Fernandez-Catalan C, Grams F, Gomis-Rüth FX, Nagase H, Tschesche $\mathrm{H}$, Maskos $\mathrm{K}$ : Insights into MMP-TIMP interactions. Ann N Y Acad Sci 1999, 878:73-9I.

23. Brew $K$, Dinakarpandian $D$, Nagase $H$ : Tissue inhibitors of metalloproteinases: evolution, structure and function. Biochim Biophys Acta 2000, I 477( I-2):267-283.

24. Holten-Andersen MN, Christensen IJ, Nielsen HJ, Lilja H, Murphy G, Jensen V, Brünner N, Piironen T: Measurement of the noncomplexed free fraction of tissue inhibitor of metalloproteinases I in plasma by immunoassay. Clin Chem 2002, 48: I305- I 3 I3.

25. Opdenakker G, Steen PE Van den, Dubois B, Nelissen I, Van Coillie E, Masure S, Proost P, Van Damme J: Gelatinase B functions as regulator and effector in leukocyte biology. J Leukoc Biol $200 \mathrm{I}$, 69:851-859.

26. Triebel S, Bläser J, Gote T, Pelz G, Schüren E, Schmitt M, Tschesche $\mathrm{H}$ : Evidence for the tissue inhibitor of metalloproteinases-I (TIMP-I) in human polymorphonuclear leukocytes. Eur J Biochem 1995, 231 :7|4-7|9.

27. Vogel I, Thorsen P, Curry A, Sandager P, Uldbjerg N: Biomarkers for the prediction of preterm delivery. Acta Obstet Gynecol Scand 2005, 84:516-525.
Publish with Bio Med Central and every scientist can read your work free of charge

"BioMed Central will be the most significant development for disseminating the results of biomedical research in our lifetime. "

Sir Paul Nurse, Cancer Research UK

Your research papers will be:

- available free of charge to the entire biomedical community

- peer reviewed and published immediately upon acceptance

- cited in PubMed and archived on PubMed Central

- yours - you keep the copyright

Submit your manuscript here:

http://www.biomedcentral.com/info/publishing_adv.asp
BioMedcentral 